\title{
Market Dynamics. On Supply and Demand Concepts.
}

\author{
Vladislav Gennadievich Malyshkin* \\ Ioffe Institute, Politekhnicheskaya 26, St Petersburg, 194021, Russia
}

(Dated: February, 14, 2016)

\$Id: SupplyDemand.tex,v 1.73 2016/02/14 06:36:58 mal Exp \$

The disbalance of Supply and Demand is typically considered as the driving force of the markets. However, the measurement or estimation of Supply and Demand at price different from the execution price is not possible even after the transaction. An approach in which Supply and Demand are always matched, but the rate $I=d v / d t$ (number of units traded per unit time) of their matching varies, is proposed. The state of the system is determined not by a price $p$, but by a probability distribution defined as the square of a wavefunction $\psi(p)$. The equilibrium state $\psi^{[H]}$ is postulated to be the one giving maximal $I$ and obtained from maximizing the matching rate functional $\left\langle I \psi^{2}(p)>/<\psi^{2}(p)>\right.$, i.e. solving the dynamic equation[1] of the form "future price tend to the value maximizing the number of shares traded per unit time". An application of the theory in a quasi-stationary case is demonstrated. This transition from Supply and Demand concept to Liquidity Deficit concept, described by the matching rate $I$, allows to operate only with observable variables, and have a theory applicable to practical problems.

\footnotetext{
* malyshki@ton.ioffe.ru
} 
Dedicated to Nastya Tabakova

\section{INTRODUCTION}

The concept of Supply \& Demand is the central concept of modern economy. With price increase the production rate increases and consumption rate decreases. The next step is to introduce the production rate (Supply curve $S(p)$ ) and the consumption rate (Demand curve $D(p))$ as two functions of price, see Fig. 1, then consider their balance $S(p)=D(p)$ as a stationary condition. However, while the statement about production and consumption rate is mostly correct, the introduction of supply $S(p)$ and demand $D(p)$ curves poses severe limitation on a type of market dynamics and have been criticized from a number of points.

Hans Albert [2], besides other problems, point to the tautology and interpretational problem with the approach, so called ceteris paribus ("all other things being equal") problem, that "... theoreticians who interpret the clause differently de facto have different laws of demand in mind, maybe even laws that are incompatible with each other." Joan Robinson [3] point to a similar problem "Utility is the quality in commodities that makes individuals want to buy them, and the fact that individuals want to buy commodities shows that they have utility". Another often discussed issues with classical type of theory is equilibrium structure, supply-demand interdependence $[4]$ and adequacy to the real world markets $[5]$.

We see the main problem with supply $S(p)$ and demand curves $D(p)$ concept that they are not measurable or even observable at price different from current. Even after the transaction is executed we can tell nothing about $S(p)$ or $D(p)$ at price different from the execution price, thus make the concept of ontological type, not applicable to practical calculations. The most intresting, the tâtonnement process [6], as a mean to observe the supply/demand curves misses the whole aspect of market dynamics[7]. In our initial approach[1] to build a dynamic theory based on observable variables the importance of execution rate $I=d v / d t$, the number of entities (e.g. equity shares) traded per unit time was emphasized, and the dynamic equation of the form "future price tend to the value maximizing the number of shares traded per unit time" was postulated and then, to some degree, observed experimentally. That paper was dealing with complicated issues of realtime HFT trading, so the calculations there were performed in P\&L space, not in price space (for a trader asset price is irrelevant, only the P\&L is important), specific time-dependent basis mixed with trading signals was 


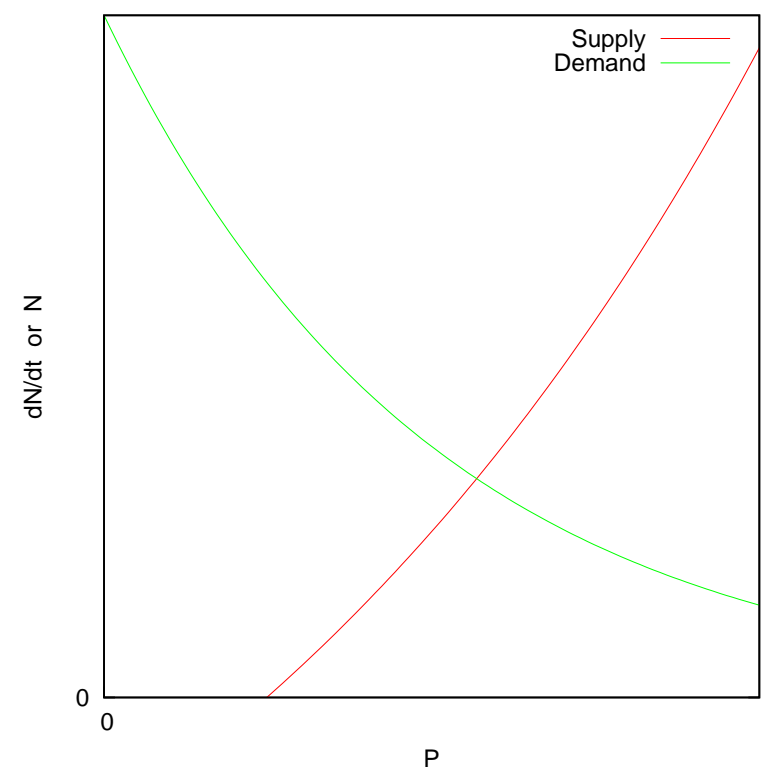

FIG. 1. Schematic plot of supply and demand as a function of price.

chosen. In this paper we consider a simplified, quasi-stationary problem, where the $I$ is assumed to be only a function of price $I(p)$, then we propose the characteristics, estimating equilibrium properties of the market. As an example US equity market for AAPL stock on the September, 20, 2012, same day used in [1] will be considered. We understand this market is actually does not have true equilibrium, but it allows us to demonstrate the technique to calculate equilibrium state from the data and show the behaviour of such characteristics as $I$, probability, and price "dynamic impact".

\section{TRADING RATE IN A QUASI-STATIONARY CASE}

As we postulated in [1] the dynamic equation has the form:

$$
\begin{gathered}
I(p) \rightarrow \max \\
I=\frac{d v}{d t}
\end{gathered}
$$

where $I$ is the number of units $d v$ traded per unit time $d t$. The $I$ describe the rate of buyers and sellers matching (a sell/buy market order matched a buy/sell limit order, already in the order book). With this definition of $I$ the buyers and the sellers are always matched. There is no any buyers-sellers disbalance at any price. What is a function of price - the rate $I$, at witch buyers and sellers match each other. Instead of using an assumption of buyers-sellers 
(demand-supply) disbalance, we consider always matched buyers-sellers and maximize the rate of matching, postulating that such a state of maximal $I$ to be the equilibrium state. In a quasi-stationary case, considered in this paper, the $I(p)$ is assumed to be a function of price only, not depending on time explicitly (this assumption is incorrect in a general case). Introduce a polynomial basis $Q_{k}(p)$ with $k=[0 . . d-1]$ (the results are invariant with respect to a linear basis transform, so as a $Q_{k}(p)$ an arbitrary polynomial of $k$-th degree can be chosen, e.g. $p^{k}$, but in practice the basis selection is determined by the numerical stability of calculations). Two measures need to be used for this theory. In the simplest form they both are a sum over all observation points, but different with $d t$ and $d v$ type of integration:

$$
\begin{aligned}
& <f>_{t}=\sum_{q}\left(t_{q}-t_{q-1}\right) f\left(t_{q}\right) \\
& <f>_{v}=\sum_{q}\left(v_{q}-v_{q-1}\right) f\left(t_{q}\right)
\end{aligned}
$$

The (3) integrate over $d t=\left(t_{q}-t_{q-1}\right)$ and the (4) integrate over $d v=\left(v_{q}-v_{q-1}\right)$, index $q$ label observation event of matching market order with limit order already in the order book. Two Gramm matrices are defined on these two measures

$$
\begin{aligned}
G_{j k}^{t} & =<Q_{j} Q_{k}>_{t}=\sum_{q}\left(t_{q}-t_{q-1}\right) Q_{j}\left(p\left(t_{q}\right)\right) Q_{k}\left(p\left(t_{q}\right)\right) \\
G_{j k}^{v} & =<Q_{j} Q_{k}>_{v}=\sum_{q}\left(v_{q}-v_{q-1}\right) Q_{j}\left(p\left(t_{q}\right)\right) Q_{k}\left(p\left(t_{q}\right)\right)
\end{aligned}
$$

The key element of the theory is an introduction of a wavefunction state $\psi(p)$, determining probability density, similar to the approach of using quantum mechanics -like probability state to describe classical measurement experiment[] $]$

$$
\psi(p)=\sum_{k=0}^{d-1} \psi_{k} Q_{k}(p)
$$

Each probability state (17) determine the value of $I$

$$
I_{\psi}=\frac{\left\langle\psi^{2}(p) I\right\rangle_{t}}{\left\langle\psi^{2}(p)\right\rangle_{t}}=\frac{\left\langle\psi^{2}(p)\right\rangle_{v}}{\left\langle\psi^{2}(p)\right\rangle_{t}}
$$

here the $\psi^{2}(p)$ can be treated as a probability density. Using Gramm matrices definitions (5) and (6) the (8) can be expressed as a ratio of two quadratic forms, estimator of a stable form[9]:

$$
I_{\psi}=\frac{\sum_{j, k=0}^{d-1} \psi_{j} G_{j k}^{v} \psi_{k}}{\sum_{j, k=0}^{d-1} \psi_{j} G_{j k}^{t} \psi_{k}}
$$


Given the (9) mapping of a $\psi$ state to the value of $I$ the question arise about the $\psi$ states of maximal $I$ (corresponding to (1) dynamic equation), of minimal $I$ (corresponding to liquidity deficit state) and of a given price value state.

The states, corresponding to minimal and maximal $I$ can be found from generalized eigenvalues problem

$$
\sum_{k=0}^{d-1} G_{j k}^{v} \psi_{k}^{[i]}=\lambda^{[i]} \sum_{k=0}^{d-1} G_{j k}^{t} \psi_{k}^{[i]}
$$

Where the $\left(\lambda^{[i]}, \psi^{[i]}(p)\right) ; i=[0 . . d-1]$ pairs define the value of $I$ and corresponding to it probability distribution $\left(\sum_{k=0}^{d-1} \psi_{k}^{[i]} Q_{k}(p)\right)^{2}$.

The $\psi^{[H]}(p)$ state, corresponding to maximal $\lambda$ (the maximal $I$ ) is special, it corresponds to the equilibrium state. This is a replacement of classical supply-demand theory where the equilibrium is determined by price, obtained from $S(p)=D(p)$ equation. In our new approach the equilibrium is defined not by a specific price $p$, but by the probability distribution $\left(\psi^{[H]}(p)\right)^{2}$ obtained as the eigenvector of (10) problem. The price, or any other observable variable, corresponding to this state, can be calculated in a similar to (8) way, e.g.

$$
p_{\psi^{[H]}}=\frac{\left\langle\left(\psi^{[H]}(p)\right)^{2} p\right\rangle_{v}}{\left\langle\left(\psi^{[H]}(p)\right)^{2}\right\rangle_{v}}
$$

The value of any observable variable in equilibrium can be calculated from (8) by replacing the $I$ by the value of interest.

A typical application of (1) dynamic equation to a quasi-stationary problem consists of calculating from observation data the $G_{j k}^{t}$ and $G_{j k}^{v}$ matrices, solving the Eq. (10) problem, obtaining the equilibrium state $\psi^{[H]}(p)$. After the $\psi^{[H]}(p)$ is found all the observable variables of interest can be calculated. An important feature of all the $\psi^{[i]}(p)$ states, including the $\psi^{[H]}(p)$, is that the first variation of $I$ from (88) on these states is equal to zero for arbitrary variation $\delta \psi(p)$, what immediately follows from the (10):

$$
\delta \frac{<\left(\psi^{[i]}(p)\right)^{2}>_{v}}{<\left(\psi^{[i]}(p)\right)^{2}>_{t}}=2\left(\frac{<\psi^{[i]}(p) \delta \psi(p)>_{v}}{<\left(\psi^{[i]}(p)\right)^{2}>_{t}}-\lambda^{[i]} \frac{<\psi^{[i]}(p) \delta \psi(p)>_{t}}{<\left(\psi^{[i]}(p)\right)^{2}>_{t}}\right)=0
$$

The second variation of $I$ from (8) on $\psi^{[i]}(p)$ states is:

$$
\delta \delta \frac{<\left(\psi^{[i]}(p)\right)^{2}>_{v}}{<\left(\psi^{[i]}(p)\right)^{2}>_{t}}=2\left(\frac{<(\delta \psi(p))^{2}>_{v}}{<\left(\psi^{[i]}(p)\right)^{2}>_{t}}-\lambda^{[i]} \frac{<(\delta \psi(p))^{2}>_{t}}{<\left(\psi^{[i]}(p)\right)^{2}>_{t}}\right)
$$


The other second variation terms vanish because of (10). For for $i=H$ the (13) is always negaive.

The variation of $p_{\psi^{[H]}}$ from (11) can be calculated by considering the $\psi^{[H]}(p)+\delta \psi(p)$ states (without losing a generality the $\delta \psi(p)$ here can be considered orthogonal to $\psi^{[H]}(p)$, i.e. $\left.\delta \psi(p)=\sum_{i=0 ; i \neq H}^{d-1} \beta^{[i]} \psi^{[i]}(p)\right)$ and the answer in the second order perturbation theory is:

$$
\begin{aligned}
p_{\psi^{[H]}+\delta \psi} & =p_{\psi^{[H]}}+\frac{2}{\lambda^{[H]}}\left\langle\psi^{[H]}(p I) \delta \psi\right\rangle_{t}+\frac{1}{\lambda^{[H]}}\left\langle\delta \psi\left(p I-p_{\psi^{[H]}} I\right) \delta \psi\right\rangle_{t} \\
& =p_{\psi^{[H]}}+\frac{2}{\lambda^{[H]}} \sum_{i=0 ; i \neq H}^{d-1} \beta^{[i]}(p I)^{[i H]}+\frac{1}{\lambda^{[H]}} \sum_{i, l=0 ; i, l \neq H}^{d-1} \beta^{[i]}\left(p I-p_{\psi^{[H]}} I\right)^{[i l]} \beta^{[l]} \\
(p I)^{[i l]} & =<\psi^{[i]}(p) p \psi^{[l]}(p)>_{v} \\
\left(p I-p_{\psi^{[H]}} I\right)^{[i l]} & =<\psi^{[i]}(p)\left(p-p_{\psi^{[H]}}\right) \psi^{[l]}(p)>_{v}
\end{aligned}
$$

The (14) is a quadratic function on $\beta^{[i]}$, and the extremum of (14) can be found by solving a linear system of $d-1$ size (the $\beta^{[H]}=0$, what reduces the size of the system by 1 ), then substituting the $\beta^{[i]}$ found back to (14) to find the value of the extremum of $p_{\psi^{[H]}+\delta \psi}$.

$$
E x\left(p_{\psi^{[H]}+\delta \psi}\right)=p_{\psi^{[H]}}-\frac{1}{\lambda^{[H]}} \sum_{i, l=0 ; i, l \neq H}^{d-1}(p I)^{[H i]}\left(\left(p I-p_{\psi^{[H]}} I\right)^{-1}\right)_{[i l]}(p I)^{[l H]}
$$

If linear system matrix (16) is degenerated this means that price variation is rather small and no information about price movement caused by execution flow spikes can be obtained. If the extremum of $p_{\psi^{[H]}+\delta \psi}$ is equal to $p_{\psi^{[H]}}$ this means that all the (15) elements $(p I)^{[i H]}=0 ; i \neq H$ vanish, what means that $I$ and $p_{\psi[H]}$ reach the extremum on the same state $\psi^{[H]}$.

The question arise of determining the state $\psi_{P}(p)$, corresponding to the specific price value $P$. In the simplest case Radon-Nikodym type of approximation[1] can be used:

$$
\psi_{P}(p)=\frac{\sum_{j, k=0}^{d-1} Q_{j}(p)\left(G^{t}\right)_{j k}^{-1} Q_{k}(P)}{\sqrt{\sum_{j, k=0}^{d-1} Q_{j}(P)\left(G^{t}\right)_{j k}^{-1} Q_{k}(P)}}
$$

Substitution of (18) to (8) give an estimate of the number of units traded per unit time at price $P$ :

$$
I(P)=\frac{\sum_{j, k, l, m=0}^{d-1} Q_{j}(P)\left(G^{t}\right)_{j k}^{-1} G_{k l}^{v}\left(G^{t}\right)_{l m}^{-1} Q_{m}(P)}{\sum_{j, k=0}^{d-1} Q_{j}(P)\left(G^{t}\right)_{j k}^{-1} Q_{k}(P)}
$$


Another important application of (18) is related to probability density analysis. The $\left(\psi^{[i]}(p)\right)^{2}$ is unbounded and is hard to scale. It is much more convenient for an analysis (and graphical representation) to use a squared projection of $\psi^{[i]}(p)$ on $\psi_{P}(p)$ :

$$
\begin{aligned}
w^{[i]}(P)= & <\psi^{[i]}(p) \psi_{P}(p)>_{t}^{2}= \\
& \frac{\left(\sum_{k=0}^{d-1} \psi_{k}^{[i]} Q_{k}(P)\right)^{2}}{\sum_{j, k=0}^{d-1} Q_{j}(P)\left(G^{t}\right)_{j k}^{-1} Q_{k}(P)}=\frac{\left(\psi^{[i]}(P)\right)^{2}}{\sum_{j, k=0}^{d-1} Q_{j}(P)\left(G^{t}\right)_{j k}^{-1} Q_{k}(P)}
\end{aligned}
$$

that is bounded to [0..1] interval. The $w^{[i]}(P)$ has a meaning of probability: how close are the two states: the $\psi_{P}(p)$, the one with price equal to $P$, and the $\psi^{[i]}(p)$, the (10) eigenstate.

\section{NUMERICAL ESTIMATION OF THE EQUILIBRIUM}

As application example of this theory let us apply it to AAPL stock data on September, 20, 2012. This data is definitely not a quasi-stationary, but let us forget about this for a moment and use the data as an illustrative example of the technique. The calculations are performed in the following way. Obtain every observation tick, labeled by $q$ index, as a triple of time,price traded, total volume since the beginning $\left(t_{q}, p_{q}, v_{q}\right)$. The volume traded at $q$-th tick is $v_{q}-v_{q-1}$ and the time passed between $q$-th and $(q-1)$-th ticks is $t_{q}-t_{q-1}$. Having

all this data available, choose a polynomial basis $Q_{k}(p)$ (the $Q_{k}(p)=p^{k}$ choice cause severe numerical instability for $d>3$, see Appendix A of [1] with a few examples of stable basis selection), and solve generalized eigenvalue problem (10) using standard, e.g. LAPACK[10] routines dsygv, dsygvd and similar. Among the eigenvectors of the (10) select the $\psi^{[H]}(p)$ corresponding to maximal $\lambda$, that give the equilibrium state probability distribution. Then all the variables of interest can be calculated from this $\psi^{[H]}(p)$.

In Fig. 2 the price of AAPL stock is presented as a function of time. This day was specifically chosen to have trending and volatility periods.

In top chart in Fig. 3 a histogram of volume distribution is presented. The sum of all histogram columns give total volume (equal to 2,630,738 shares). reported by NASDAQ ITCH feed between 9:30 and 16:00 on September, 20, 2012. (Total AAPL traded shares reported by ITCH feed on that day, including off-market hours, is 3,063,928 ; Google Finance $(84,141,932)$ \& Yahoo Finance $(84,142,100)$ report higher volume, as consolidated from a 


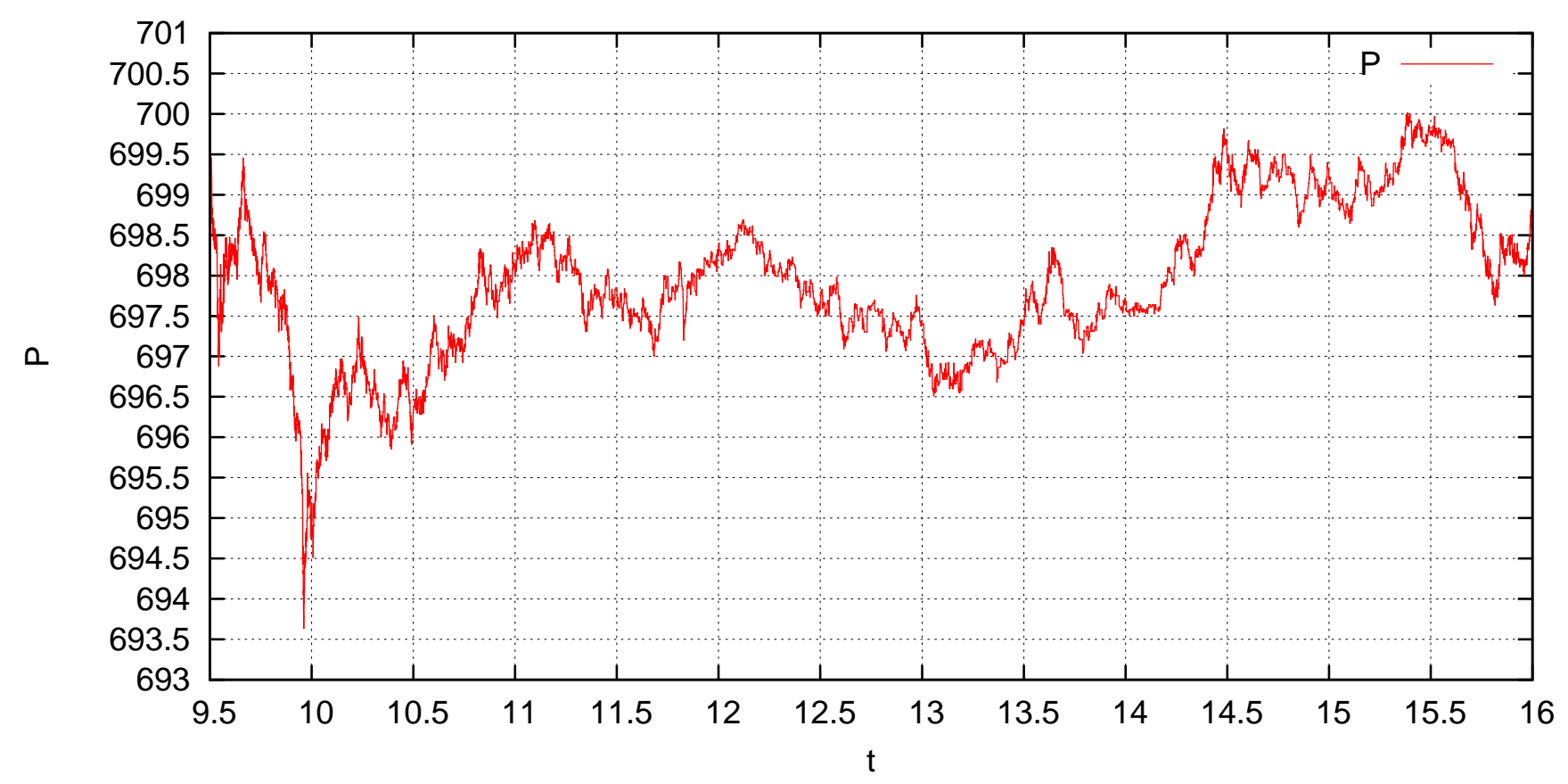

FIG. 2. The AAPL stock price on September, 20, 2012. The time on $\mathrm{x}$ axis is in decimal fraction of an hour, e.g. 9.75 mean 9:45am.

number of exchanges). Price analysis of various kinds, e.g. skewness, fat tails analysis, etc. is often performed by analysts considering the charts similar to this price-volume distribution. We want to stress, that the volume distribution carry no information about the dynamics. First, this distribution assume some manually selected time interval for the analysis. This make the result observer-dependent. Second, maximization of trading volume is equivalent to "buy below median price" and "sell above median price" type of trading strategy. This type of strategy assume the median price, that is a non-local value (depend on the entire observation set), is known, what is not the case for market dynamics, that is determined by the events of a local nature on relatively small (and unknown upfront) time scales.

In the low charts in Fig. 3 the results for the theory are presented for $d=10$ elements in basis. In the second chart in Fig. 3 the $I(P)$ at given price $P$ is calculated using the state (18) to receive (19). One can see a much sharper picture, than the one with the volume histogram. There are high values of $I$ near the 694 and 700 price levels. These levels correspond to a very active trading (large $I$ ) at around 10am and near market close at 15:30. The theory automatically select the price levels of high trading activity, not the high total volume traded as in the chart above, that provide volume median at about the 698 level. 

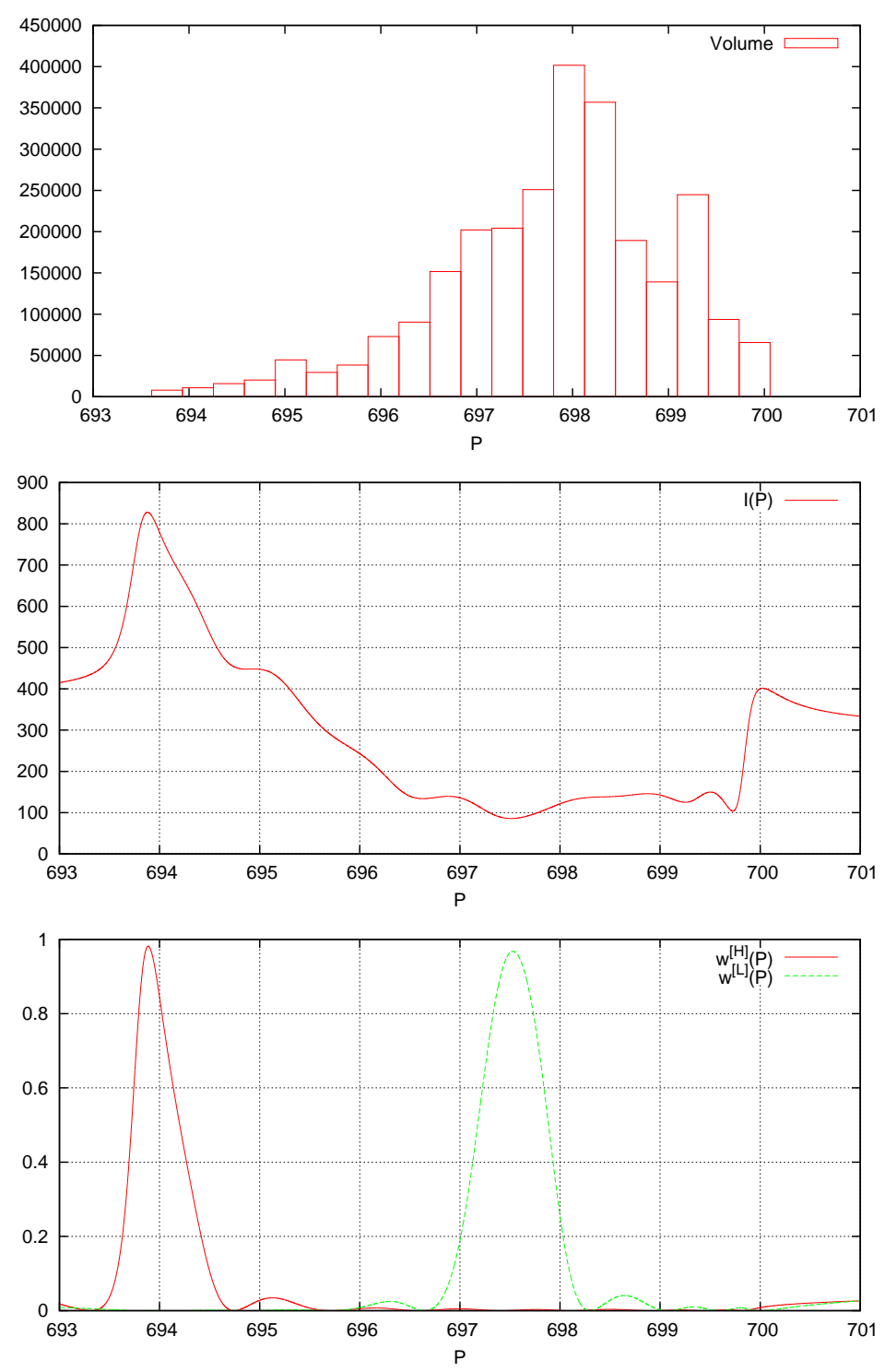

FIG. 3. Price histogram for volume distribution. $I(P)$ from (19) as a function of price. Projections (20) to the states corresponding to highest $w^{[H]}(P)$ and lowest $w^{[L]}(P)$ value of $I$.

Most of the shares were actually traded near this, close to median, price.

There is no singularity in total volume distribution near 694 and 700 price levels, because despite a high value of $I$ the total time spent at these price levels is low, what result in low volume traded. But in terms of price change, risk analysis and market dynamics the volume per unit time, not the total volume should be used, because this characteristics describe market activity at given price level. From these two charts (volume distribution and $I(P))$ we can make an important observation on price impact. The price impact [11 13$]$ is typically considered as path-dependent impact of executed shares on asset price. As we 
see from the volume distribution chart the volume near 694 level is rather low, but the price change and $I$ are high. This make reasonable to introduce a "dynamic impact" concept, the sensitivity of asset price to the rate $I$, different from regular "impact" — the sensitivity of asset price to executed volume [14]. From the charts presented we see an importance of the "dynamic impact" concept, as both price and $I$ singularities are localized at about the same price levels. Similar situation occur in the time-space, [1] what make one more argument, that price change is related to $I$, not to the volume and the "dynamic impact" should be considered as the major contribution of trading activity affecting asset price. The Eq. (17) give an opportunity to experimentally answer on the fundamental question whether the extremum of $I$ matches the extremum of $p_{\psi[H]}$. In classical impact [14] model (increase in trading volume causes substantial price changes) the extremums of $I$ and $p_{\left.\psi^{[H]}\right]}$ should be substantially different. In the "dynamic impact" model (an increase in I causes substantial price changes) the extremums of $I$ and $p_{\psi^{[H]}}$ correspond the same state $\psi^{[H]}$. For the data we used the values $p_{\psi^{[H]}}=693.96$ and $E x\left(p_{\psi^{[H]}}\right)=692.46$ were obtained. This, along with observation of large price movement near large $I$ make the "dynamic impact" model seems to be more appropriate. However, the wavefunction in price space are applicable only to a quasistationary case and different basis need to be considered when trying to make a conclusion about a relative importance of "classical impact" and "dynamic impact" in a non-stationary case.

The transition from total volume (number of shares) to volume traded per unit time allows to overcome two mentioned above limitations of price-volume analysis. First, in contrast with price median, that has no degree of freedom, and is determined only by the manually inserted time scale, the (10) eigenvalues problem have the $d$ degrees of freedom, what allows automatic selection of the most appropriate time scale according to the dynamic equation (1). Second, in contrast with the total volume traded the trading rate $I=d v / d t$ is local, a few large trade executed in a short time period can drastically change the value of $I$. One can see this transition from volume to volume per unit time as some kind similar to the transition of momentum of motion concept from Aristotle to Galilei type of classical mechanics.

In the third chart in Fig. 3 we present the probabilities $w^{[H]}(P)$ and $w^{[L]}(P)$ of a given price $P$ to correspond to the state of maximal and minimal $I$ respectively. The $w^{[H]}(P)$ is localized in the area of high $I$ and large price changes (the "dynamic impact" effect). 
The $w^{[L]}(P)$ is localized in the area of low $I$, in a quasi-stationary case the probability $w^{[L]}(P)$ has no importance, but it is extremely important in a non-stationary case 1$]$, as determining trading position opening condition. Market equilibrium interpretation is now have a probabilistic nature and is determined by a wavefunction $\psi^{[H]}(p)$ of the equilibrium state, corresponding to maximal $I$. This is a major transition from price-space classical equilibrium $S(P)=D(P)$ to the dynamic equation of (1) form. The goal of this transition is to obtain the equilibrium state directly from market data and, as we demonstrated above with the $\psi^{[H]}(p)$ calculation, this can performed by solving the (10) problem. This is a critical step forward compared to the estimation of classical supply $S(P)$ and demand $D(P)$ functions from market data, what is ambiguous at best and impossible at worst. The transition from supply/demand to matching rate shift the study of $S(P)=D(P)$ in the vicinity of equilibrium (e.g. whether it has linear or square root type of behavior[7]) to the study of $I$ in the vicinity of $\psi^{[H]}(p)$, because, as we demonstrated experimentally (see Appendix A for code and data), the dynamic changes in price due to $I$ changes are much greater than price changes due to volume changes. In this paper only quasi-stationary case is considered, what lead to only two types of $I$ variations: Eq. (12) and (13), with $\delta \psi(p)$ as the $\psi^{[H]}(p)$ variation in price space. For the full dynamic theory in our earlier work[1] we considered a probability state in time space $\psi(t)$ with two measures(Laguerre and Shifted Legendre) form Section II "Kinematics" of Ref. [1] having extremely convenient properties that allows to obtain infinitesimal time-shift variation by applying integration by parts. The question arise about combining the $\psi(t)$ and $\psi(p)$ probability states into a single state, that would describe price dynamics, and give an answer of how much information about future price values can be actually obtain, i.e. to what extend a trader dream of "philosopher stone", the system that predict future prices, can actually exist. This would be the topic of our future research.

\section{DISCUSSION}

In this paper an alternative to Supply-Demand theory is proposed. The theory is considering always matched buyers and sellers, and maximizing the rate of their matching. System state is determined by a probability distribution, from which all observable variable, including matching rate, can be calculated. The equilibrium distribution, corresponding to 
maximal matching rate, can be found from generalized eigenvalues problem, maximizing the matching rate functional (9). An application of the theory is demonstrated on AAPL intraday trading data. A conceptual difference between maximizing the trading volume and matching rate (trading volume per unit time) is shown. While the trading volume has maximal values about median price, the matching rate has a singularity-like behavior near the market tipping points, what make the approach much more suitable to risk measurement and market direction prediction.

\section{Appendix A: Code implementation example}

Computer code implementing the algorithms is available[15]. The code is java/scala written. To reproduce the results follow these steps:

- Install java 1.8 or later \& scala 2.11 .7 or later.

- Download from [15] the data file S092012-v41.txt.gz and code archive SupplyDemandQuasiStationary.zip.

- Decompress the code and recompile it.

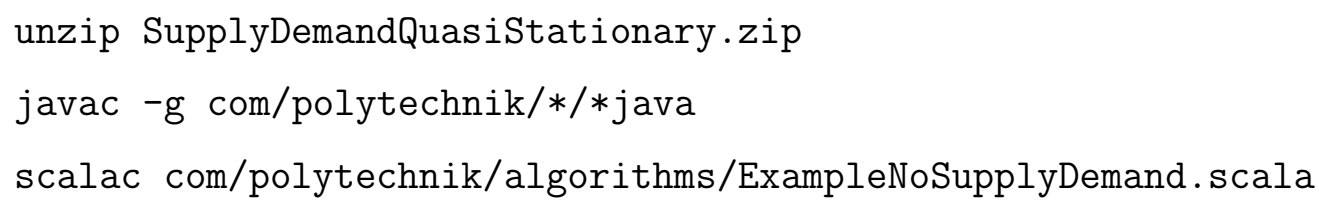

- Extract from the file S092012-v41.txt.gz AAPL executed trades and save the data to aapp.csv

java com/polytechnik/itch/DumpDataTrader S092012-v41.txt.gz AAPL >aapl.csv

The $P(t)$ in Fig. 2 can be obtained from the aapl.csv, the unit of time (second column) in aapl.csv is the one used in NASDAQ ITCH[16]: nanoseconds since 00:00.

- Use executed trades information from aapl.csv to obtain Section III results.

scala com.polytechnik.algorithms.ExampleNoSupplyDemand aapl.csv 
The files histogram.csv and psi.csv are now generated. The file histogram.csv contains price-volume distribution (top chart in Fig. 3). The file psi.csv contains $w^{[H]}(P)$, $w^{[L]}(P)$ and $I(P)$ (other charts in Fig. 3).

[1] Vladislav Gennadievich Malyshkin and Ray Bakhramov, "Mathematical Foundations of Realtime Equity Trading. Liquidity Deficit and Market Dynamics. Automated Trading Machines. http://arxiv.org/abs/1510.05510," ArXiv e-prints (2015), arXiv:1510.05510 [q-fin.CP],

[2] Hans Albert, Darrell Arnold, and Frank Maier-Rigaud, "Model platonism: Neoclassical economic thought in critical light," Journal of Institutional Economics 8, 295-323 (2012).

[3] Joan Robinson, Economic philosophy (Transaction Publishers, 1962).

[4] Alan Kirman, "The intrinsic limits of modern economic theory: the emperor has no clothes," The Economic Journal , 126-139 (1989).

[5] Neva Goodwin, Julie A Nelson, Frank Ackerman, and Thomas Weisskopf, "Microeconomics in context," New York (2009), ISBN:978-0-7656-2301-0.

[6] Leon Walras, Elements of pure economics (Routledge, 2013).

[7] Jonathan Donier and Jean-Philippe Bouchaud, "From Walras' auctioneer to continuous time double auctions: A general dynamic theory of supply and demand," ArXiv e-prints (2015), arXiv:1506.03758 [q-fin.TR].

[8] Vladislav Gennadievich Malyshkin, "Norm-Free Radon-Nikodym Approach to Machine Learning," arXiv preprint arXiv:1512.03219 (2015).

[9] Gennadii Stepanovich Malyshkin, Optimal and Adaptive Methods of Hydroacoustic Signal Processing. Vol 1. (Elektropribor Publishing, 2009) ISBN: 978-5-900780-90-0.

[10] "Lapack version 3.5.0,"] (2013).

[11] E. Moro, J. Vicente, L. G. Moyano, A. Gerig, J. D. Farmer, G. Vaglica, F. Lillo, and R. N. Mantegna, "Market impact and trading profile of hidden orders in stock markets," Phys. Rev. E 80, 066102 (2009), arXiv:0908.0202 [q-fin.TR],

[12] Jim Gatheral and Alexander Schied, "Dynamical models of market impact and algorithms for order execution," HANDBOOK ON SYSTEMIC RISK, Jean-Pierre Fouque, Joseph A. Langsam, eds , 579-599 (2013). 
[13] J. Donier, J. Bonart, I. Mastromatteo, and J.-P. Bouchaud, "A fully consistent, minimal model for non-linear market impact," ArXiv e-prints (2014), arXiv:1412.0141 [q-fin.TR],

[14] Wikipedia, "Market impact,"] (2016).

[15] Vladislav Gennadievich Malyshkin, a (2014), the code for polynomials calculation, http://www.ioffe.ru/LNEPS/malyshkin/code.html

[16] Nasdaq OMX, NASDAQ TotalView-ITCH 4.1, Report (Nasdaq OMX, 2014). 